\title{
Effectiveness and cost-effectiveness of double reading in digital mammography screening: A systematic review and meta-analysis(Article)
}

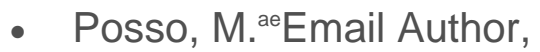

- Puig, T. ${ }^{\mathrm{ab}}$ Email Author,

- Carles, M.'Email Author,

- Rué, M. 'Email Author,

- Canelo-Aybar, C. ${ }^{\text {efEmail Author, }}$

- Bonfill, X. ${ }^{\text {abeg} E m a i l ~ A u t h o r ~}$

- View Correspondence (jump link)

- $\quad$ aDepartment of Clinical Epidemiology and Public Health, Hospital de la Santa Creu i Sant Pau (IIB Sant Pau), Barcelona, Spain

- $\quad$ 'Universitat Autònoma de Barcelona (UAB), Barcelona, Spain

- $\quad$ Economics Department and CREIP, Universitat Rovira i Virgili, Reus, Spain

View additional affiliations_

Abstract_View references (32)

Purpose Double reading is the strategy of choice for mammogram interpretation in screening programmes. It remains, however, unknown whether double reading is still the strategy of choice in the context of digital mammography. Our aim was to determine the effectiveness and cost-effectiveness of double reading versus single reading of digital mammograms in screening programmes. Methods We performed a systematic review by searching the PubMed, Embase, and Cochrane Library databases up to April 2017. We used the QUADAS-2 (Quality Assessment of Diagnostic Accuracy Studies) tool and CHEERS (Consolidated Health Economic Evaluation Reporting Standards) checklist to assess the methodological quality of the diagnostic studies and economic evaluations, respectively. A proportion's metaanalysis approach, $95 \%$ Confidence Intervals $(95 \% \mathrm{Cl})$ and test of heterogeneity $(\mathrm{P}$ values) were used for pooled results. Costs are expressed US\$ PPP (United States Dollar purchasing power parities). The PROSPERO ID of this Systematic Review's protocol is CRD42014013804. Results Of 1473 potentially relevant hits, four high-quality studies were included. The pooled cancer detection rate of double reading was 6.01 per 1000 screens ( $\mathrm{Cl}: 4.47 \%-7.77 \%$ ), and it was 5.65 per 1000 screens $(\mathrm{Cl}: 3.95 \% 0-7.65 \%$ ) for single reading $(P=0.76)$. The pooled proportion of false-positives of double reading was 47.03 per 1000 screens (Cl: 39.13\%o$55.62 \%$ ) and it was 40.60 per 1000 screens (Cl: $38.58 \% 0-42.67 \%$ ) for single reading $(P=0.12)$. One study reported, for double reading, an ICER (Incremental Cost-Effectiveness Ratio) of 16,684 Euros (24,717 US\$ PPP; 2015 value) per detected cancer. Single reading + CAD (computer-aided-detection) was cost- 
effective in Japan. Conclusion The evidence of benefit for double reading compared to single reading for digital mammography interpretation is scarce.

Double reading seems to increase operational costs, have a not significantly higher false-positive rate, and a similar cancer detection rate. (C) 2017 Elsevier B.V.

Author keywords

- Breast neoplasms

- Cost and cost analysis

- Mammography

- Mass screening

- Systematic review

- ISSN: 0720048X

- CODEN: EJRAD

- Source Type: Journal

- Original language: English

- DOI: 10.1016/j.ejrad.2017.09.013

- Document Type: Article

- Publisher: Elsevier Ireland Ltd 